\title{
Activity of Antibiotics against Pseudomonas aeruginosa in an In Vitro Model of Biofilms in the Context of Cystic Fibrosis: Influence of the Culture Medium
}

\author{
Yvan Diaz Iglesias, ${ }^{a}$ (D) Françoise Van Bambeke ${ }^{a}$ \\ aPharmacologie cellulaire et moléculaire; Louvain Drug Research Institute, Université catholique de Louvain (UCLouvain), Brussels, Belgium
}

\begin{abstract}
Pseudomonas aeruginosa is a major cause of respiratory biofilm-related infections in patients with cystic fibrosis. We developed an in vitro pharmacodynamic model to study the activity of antipseudomonal antibiotics against PAO1 biofilms grown in artificial sputum medium with agar $[\mathrm{ASM}(+)]$ versus that against biofilms grown in Trypticase soy broth supplemented with glucose and $\mathrm{NaCl}$ (TGN). We measured bacterial counts, metabolic activity (fluorescein diacetate [FDA] hydrolysis), and biomass (crystal violet absorbance). Biofilms grew slower in ASM(+) than in TGN but reached the same CFU counts and metabolic activity in both media and a slightly higher biomass after $48 \mathrm{~h}$ in $\mathrm{ASM}(+)$ than in TGN. The concentration-response curves of the antibiotics after $24 \mathrm{~h}$ of incubation with mature biofilms showed maximal effects ranging from a 3 (ciprofloxacin)- to a 1.5 (ceftazidime, meropenem)- $\log _{10}$-CFU decrease, with tobramycin and colistin showing intermediate values. These maximal reductions in the numbers of CFU were similar in both media for ciprofloxacin and $\beta$-lactams but lower in $\mathrm{ASM}(+)$ than in TGN for tobramycin and colistin; they were reached at concentrations lower than the human maximum concentration in plasma for ciprofloxacin and $\beta$-lactams only. The reductions in metabolic activity and in biomass were low in both media. Small-colony variants were selected by tobramycin in $\operatorname{ASM}(+)$ and by ciprofloxacin in both media. The model was then successfully applied to 4 isolates from patients with cystic fibrosis. These biofilms showed CFU counts similar to those of PAO1 biofilms in $\mathrm{ASM}(+)$ but a higher biomass than PAO1 biofilms in $\mathrm{ASM}(+)$ and moderate differences in their susceptibility to antibiotics from that of PAO1 biofilms grown in this medium. This model proved useful to establish the pharmacodynamic profile of drugs against $P$. aeruginosa biofilms in the context of cystic fibrosis.
\end{abstract}

KEYWORDS Pseudomonas aeruginosa, antibiotic, artificial sputum medium, biofilms, ceftazidime, ciprofloxacin, colistin, cystic fibrosis, meropenem, tobramycin

seudomonas aeruginosa is the most prevalent microorganism in the respiratory tract of adult patients suffering from cystic fibrosis (CF), being detected in $70 \%$ of adults aged 25 years or older (1). It is a major cause of mortality in this population thanks to its capacity to colonize the sputum, where it forms microcolonies embedded in an alginate matrix, strongly suggestive of biofilms (2). Biofilms are indeed defined as microbial communities living in a self-produced matrix essentially composed of polysaccharides, extracellular DNA, and proteins (3-5). These structures offer a protection against host defenses and antibiotics due to the barrier effect of the extracellular matrix as well as to metabolic changes in the bacterial populations, leading to dormant phenotypes less responsive to antibiotics $(3,6)$.

One of the determining factors in the capacity of $P$. aeruginosa to colonize the lungs and form biofilms therein is the accumulation of a thickened mucus as a consequence of the dysfunction of the cystic fibrosis transmembrane conductance regulator channel
Citation Diaz Iglesias Y, Van Bambeke F. 2020 Activity of antibiotics against Pseudomonas aeruginosa in an in vitro model of biofilms in the context of cystic fibrosis: influence of the culture medium. Antimicrob Agents Chemother 64:e02204-19. https://doi.org/10 .1128/AAC.02204-19.

Copyright $\odot 2020$ American Society for Microbiology. All Rights Reserved. Address correspondence to Françoise Van Bambeke, francoise.vanbambeke@uclouvain.be Received 3 November 2019 Returned for modification 7 December 2019 Accepted 24 January 2020

Accepted manuscript posted online 3

February 2020

Published 24 March 2020 
A Influence of time on FDA metabolisation by planktonic cultures at increasing $O D_{620 \mathrm{~nm}}$

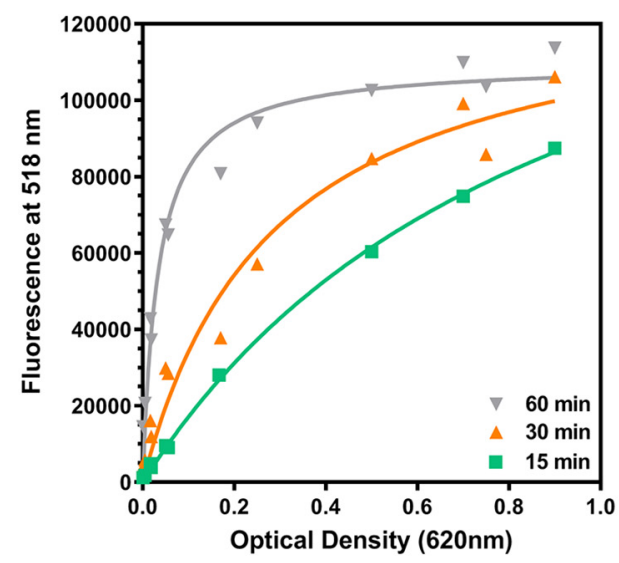

B metabolisation of FDA by planktonic cultures as a function of bacterial counts

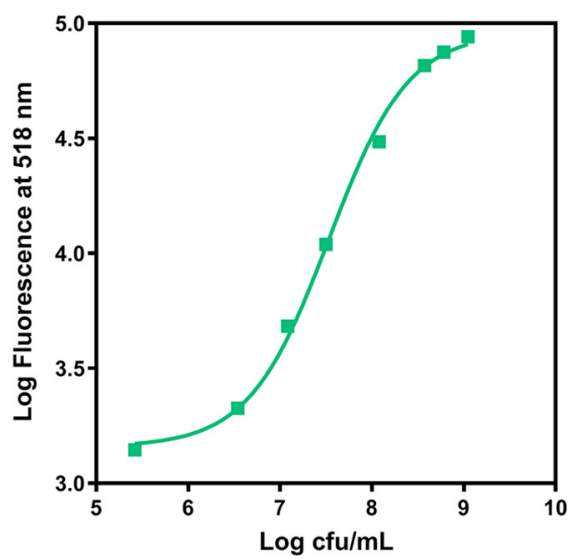

FIG 1 Establishing experimental conditions for fluorescein assay. (A) Fluorescein fluorescence signal measured for planktonic cultures of PAO1 at increasing $\mathrm{OD}_{620}$ values after 15, 30, and $60 \mathrm{~min}$ of incubation with 100-mg/liter fluorescein diacetate (FDA). (B) Correlation between the fluorescence signal measured after 15 min of incubation and CFU counts. Data are means \pm SD from 3 independent determinations.

and the subsequent impaired mucociliary clearance of inhaled microbes $(7,8)$. In addition, $P$. aeruginosa may display a large variety of phenotypes, like mucoid or small-colony variants (SCVs). The latter can be selected by antibiotics, which inhibit their growth, adding another risk factor for both the persistence of the infection $(9,10)$ and biofilm formation, as SCVs overproduce matrix polysaccharides $(5,9,10)$.

In spite of the clinical importance of these infections, only a few studies have examined in detail the activity of antibiotics in a medium that mimics the sputum of patients with CF (11-13). In the present study, we used a previously described medium (artificial sputum medium [ASM] with agar $[\mathrm{ASM}(+)]$ ) that mimics much better the composition and rheological properties of the sputum of CF patients (14) than the so-called synthetic CF sputum medium (SCFM) (15). An improved version of SCFM containing major constituents of sputum, like DNA, $N$-acetyl-D-glucosamine, mucin, and dioleoylphosphatidylcholine, could also have been used (16), but its rheological properties have not been established so far. We study the activity of antibiotics in vitro against biofilms made by $P$. aeruginosa in this medium and on a pharmacodynamic basis, in order to establish their relative potency and maximal efficacy (17). We systematically compared ASM(+) with Trypticase soy broth (TSB) supplemented by glucose and $\mathrm{NaCl}$ (TGN), a medium optimized to favor the growth of biofilms of $S$. aureus and other bacterial species (17; unpublished data from our team). We selected 5 antibiotics frequently used in these patients, namely, the fluoroquinolone ciprofloxacin, the $\beta$-lactams ceftazidime and meropenem, the aminoglycoside tobramycin, and the polymyxin colistin (6).

\section{RESULTS}

Establishing experimental conditions for FDA assay with PA01. As a preliminary experiment, we determined the optimal conditions for assaying bacterial metabolic activity using the fluorescein diacetate (FDA) assay, in which FDA is cleaved into fluorescein by bacterial enzymes produced by metabolically active bacteria. To this end, we examined the influence of the time of incubation with FDA on fluorescein fluorescence for planktonic cultures of PAO1 at different inocula in cation-adjusted MuellerHinton broth (MHB-Ca). The numbers of CFU were measured in parallel. Figure $1 \mathrm{~A}$ shows the evolution of the fluorescence signal as a function of the optical density at $620 \mathrm{~nm}\left(\mathrm{OD}_{620}\right)$ at 3 different incubation times. After $15 \mathrm{~min}$ of incubation, the fluorescence signal increased almost linearly ( $R^{2}$ of the linear regression, 0.986) for $\mathrm{OD}_{620}$ values of $\leq 0.5$ (corresponding to approximately $4 \times 10^{8} \mathrm{CFU} / \mathrm{ml}$ ), after which a 
Viability

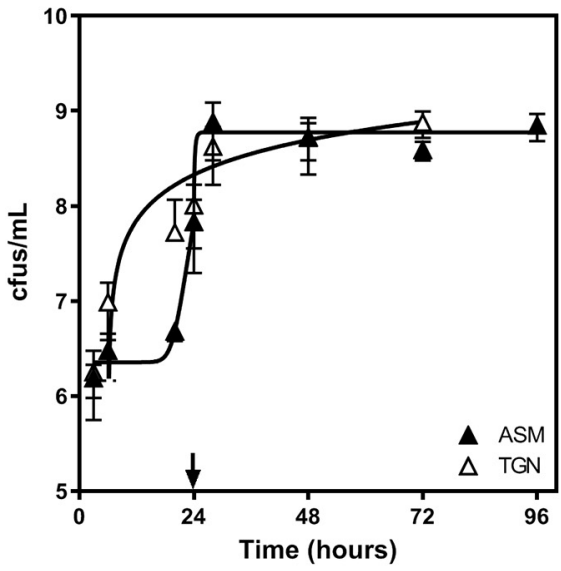

Metabolic activity

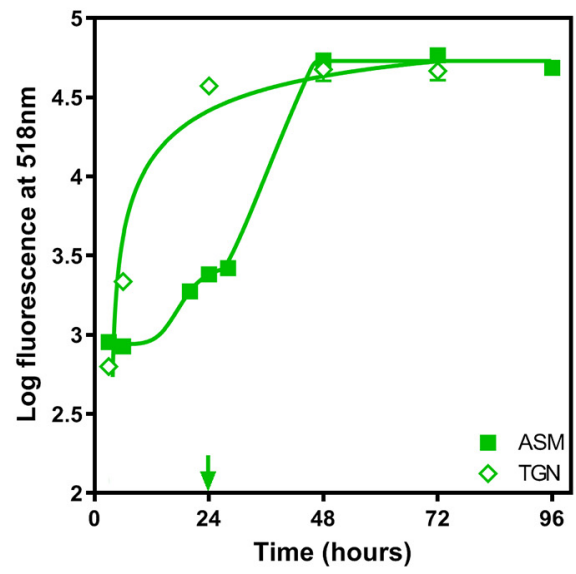

Biomass

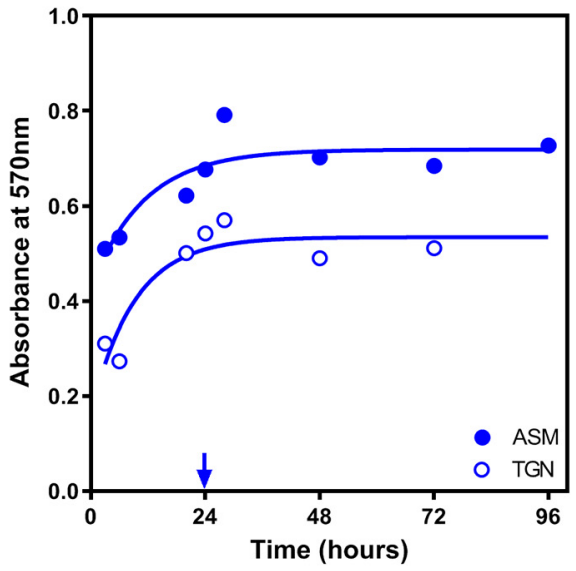

FIG 2 Kinetics of growth of biofilm in TGN and ASM. The initial inocula were $3 \times 10^{7} \mathrm{CFU} / \mathrm{ml}$ in TGN and $5.5 \times 10^{7}$ in ASM(-). In this case, attachment was allowed for $24 \mathrm{~h}$ in $\mathrm{ASM}(-)$, after which the medium was replaced by $\mathrm{ASM}(+)$ (highlighted by arrows on the $x$ axes). The graphs show the evolution over time of the numbers of CFU (viability), the fluorescein fluorescence generated by the hydrolysis of fluorescein diacetate (FDA) by metabolically active bacteria (metabolic activity or vitality), or the crystal violet absorbance (biomass). Data are means \pm SD from at least 3 independent experiments performed in 3 replicates. When not visible, the SD were smaller than the size of the symbols.

trend to a saturation of the signal was observed. When the time of incubation was prolonged to 30 or $60 \mathrm{~min}$, the range over which the relationship between the $\mathrm{OD}_{620}$ and fluorescence was linear was restricted to $\mathrm{OD}_{620}$ values of $<0.17$ (corresponding to approximately $10^{8} \mathrm{CFU} / \mathrm{ml}$ ). Figure $1 \mathrm{~B}$ shows the relationship between the fluorescence signal and bacterial counts after $15 \mathrm{~min}$ of incubation. This relationship was linear over counts ranging from $10^{6.5}$ to $10^{8.5} \mathrm{CFU} / \mathrm{ml}$ ( $R^{2}$ of the linear regression, 0.9985 ), covering the numbers recovered from biofilms (see below). This time of incubation was therefore adopted for all experiments.

Setting up the biofilm model with PA01. The kinetics of growth of PAO1 in a biofilm was followed over $72 \mathrm{~h}$ in TGN and ASM with agar $[\mathrm{ASM}(+)]$. Preliminary experiments showed that PAO1 was unable to grow and form a biofilm if it was directly inoculated in $\operatorname{ASM}(+)$, which we attributed to the high viscosity of this medium. We therefore incubated the bacteria first in ASM without agar [ASM(-)] for $24 \mathrm{~h}$ to allow for adhesion, after which the medium was replaced by $\operatorname{ASM}(+)$. Figure 2 shows the evolution of the numbers of CFU (left); metabolic activity, as assessed by the FDA assay (middle); or biomass, as evaluated by crystal violet staining (right), over time. In TGN, the bacterial counts increased from $10^{7} \mathrm{CFU} / \mathrm{ml}$ to $7 \times 10^{8} \mathrm{CFU} / \mathrm{ml}$ over the first $24 \mathrm{~h}$, after which a plateau was reached. Fluorescein fluorescence and crystal violet absorbance increased in parallel. In ASM, bacterial growth was minimal during the first day of incubation in $\operatorname{ASM}(-)$, after which it rapidly recapitulated to reach a plateau at $7 \times$ $10^{8} \mathrm{CFU} / \mathrm{ml}$ in $8 \mathrm{~h}$. The fluorescence signal followed a similar pattern, with, however, a slow increase in the fluorescence signal being seen in the first hours following the change of medium from $\operatorname{ASM}(-)$ to $\operatorname{ASM}(+)$. The biomass also increased over time at a rate similar to that observed in TGN but with slightly higher values all over the time of incubation. On this basis, an incubation time of $24 \mathrm{~h}$ in TGN and of $24 \mathrm{~h}$ in ASM(-) followed by $24 \mathrm{~h}$ in $\mathrm{ASM}(+)$ was considered to generate a stable biofilm (with respect to bacterial counts, metabolic activity, and biomass) that could be exposed to antibiotics.

Antibiotic activity against planktonic cultures of PA01. Table 1 shows the MICS of the selected antibiotics against PAO1 in TGN and $\mathrm{ASM}(+)$ media compared to those in cation-adjusted Mueller-Hinton broth (MHB-ca). PAO1 was fully susceptible to all antibiotics tested. In TGN, MICs were similar ( \pm 1 doubling dilution) to those in MHB-ca for all antibiotics except tobramycin, the MIC of which was 8-fold (3 doubling dilutions) higher in TGN. The MICs were 2-fold (1 doubling dilution) lower in $A S M(+)$ than in 
TABLE 1 Antibiotic susceptibility of PAO1 in different media

\begin{tabular}{|c|c|c|c|c|c|}
\hline \multirow[b]{2}{*}{ Antibiotic } & \multicolumn{3}{|c|}{ MIC (mg/liter) } & \multirow{2}{*}{ 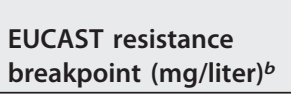 } & \multirow{2}{*}{$\begin{array}{l}\text { Human } C_{\text {max }} \\
\text { (mg/liter) }^{c}\end{array}$} \\
\hline & MHB-ca & TGN & $\operatorname{ASM}(+)^{a}$ & & \\
\hline Ciprofloxacin & 0.125 & 0.125 & 0.06 & 0.5 & 3.7 \\
\hline Ceftazidime & 1 & 0.5 & 0.25 & 8 & $87-170$ \\
\hline Meropenem & 0.5 & 0.5 & 0.125 & 8 & $50-115$ \\
\hline Tobramycin & 0.25 & 2 & 0.125 & 4 & $16-30$ \\
\hline Colistin & 0.5 & 0.5 & 2 & 2 & 2.7 \\
\hline
\end{tabular}

${ }^{a} \mathrm{MIC}$ determined by counting of the number of CFU after plating due to the turbidity of the medium. ${ }^{b} \mathrm{MIC}$ value above which the strain is considered as resistant to the tested antibiotic.

cBased on the Belgian summary of product characteristics (prescribing information) for each drug at the conventional and high (as recommended for $P$. aeruginosa infections) registered doses or based on reference 48 for colistin.

MHB-ca for ciprofloxacin and tobramycin and 4-fold (2 doubling dilutions) lower for $\beta$-lactams but 4-fold (2 doubling dilutions) higher for colistin.

Antibiotic activity against biofilms of PA01. The activities of these five antibiotics against bacterial viability, vitality (metabolic activity), and biofilm biomass were then evaluated after $24 \mathrm{~h}$ of incubation of stable biofilms in TGN or in $\mathrm{ASM}(+)$, defined as biofilms precultivated for $24 \mathrm{~h}$ in TGN or for $24 \mathrm{~h}$ in $\mathrm{ASM}(-)$ followed by $24 \mathrm{~h}$ in $\mathrm{ASM}(+)$, respectively. The corresponding concentration-response curves are presented in Fig. 3, and the pertinent calculated pharmacodynamic parameters (the maximal effect $\left[E_{\text {max }}\right]$ extrapolated for an infinitely large concentration or the concentration needed to achieve a reduction in the number of CFU of $1 \log _{10}$ [viability] $\left[C_{-1} \mathrm{log}\right.$, which corresponds to the concentration needed for a $0.1-\log _{10}$ reduction in fluorescein fluorescence [metabolic activity] $\left[C_{-0.1}\right]$ or a $0.3-\log _{10}$ decrease in the crystal violet $\mathrm{OD}_{570}$ [biomass] $\left[C_{-0.3}\right]$; see reference 14 for more explanations of these parameters) are presented in Fig. 4.

Considering antibiotic effects on CFU first, we observed a concentration-dependent reduction in all cases, which could be fitted to sigmoidal regressions. Minor differences between the two media were observed for all antibiotics except tobramycin and colistin, which were more active in TGN than in $\mathrm{ASM}(+)$ (Fig. 4A, left). The maximal effect ranged from a $3-\log _{10}$-CFU decrease for ciprofloxacin, which was the most effective, to a 1.5 - to $1.8-\log _{10}$-CFU decrease for $\beta$-lactams, which were the least effective. For tobramycin and colistin, the maximal effect ranged from a 2 - and 3 - $\log _{10}$-CFU decrease (note that a plateau value was not reached at the highest concentration tested in these cases, so that we rather report the effect observed at the highest concentration tested [100 mg/liter]). This $E_{\max }$ was obtained at clinically achievable concentrations (lower than the maximum concentration in plasma $\left[C_{\max }\right]$ in humans) for ciprofloxacin and $\beta$-lactams but not for tobramycin and colistin (see the dashed red lines in Fig. 3, which point to $C_{\max }$ values). The concentrations needed to reduce the inoculum by $1 \log _{10}$ (Fig. 4B, left) were close to the MIC in TGN for ciprofloxacin and $\beta$-lactams but higher than the MIC for tobramycin and colistin in TGN and for all drugs in $\operatorname{ASM}(+)$. They were lower than the human $C_{\max }$ in both media for ciprofloxacin and $\beta$-lactams, close to this value for tobramycin, but higher for colistin. We noticed the presence of small colonies on plates from biofilms incubated with ciprofloxacin in both media or with tobramycin in $\operatorname{ASM}(+)$, the proportion of which increased with the antibiotic concentration (Fig. 5). We therefore compared the CFU counts obtained under these conditions after $24 \mathrm{~h}$ and $48 \mathrm{~h}$ of incubation of Pseudomonas isolation agar (PIA) plates (black and gray curves, respectively, on the corresponding graphs in Fig. 3) but did not find any difference in the global reduction in bacterial counts, suggesting the we did not underestimate the CFU counts after $24 \mathrm{~h}$, despite the small size of some of them.

Considering, then, the effects of antibiotics on metabolic activity, we observed globally low reductions in the fluorescence signal (compared to those observed in planktonic cultures for equivalent reductions in CFU counts [a $0.1-\log _{10}$ difference versus a $0.8-\log _{10}$ difference in the fluorescence signal over a $1-\log _{10}$ change in the 

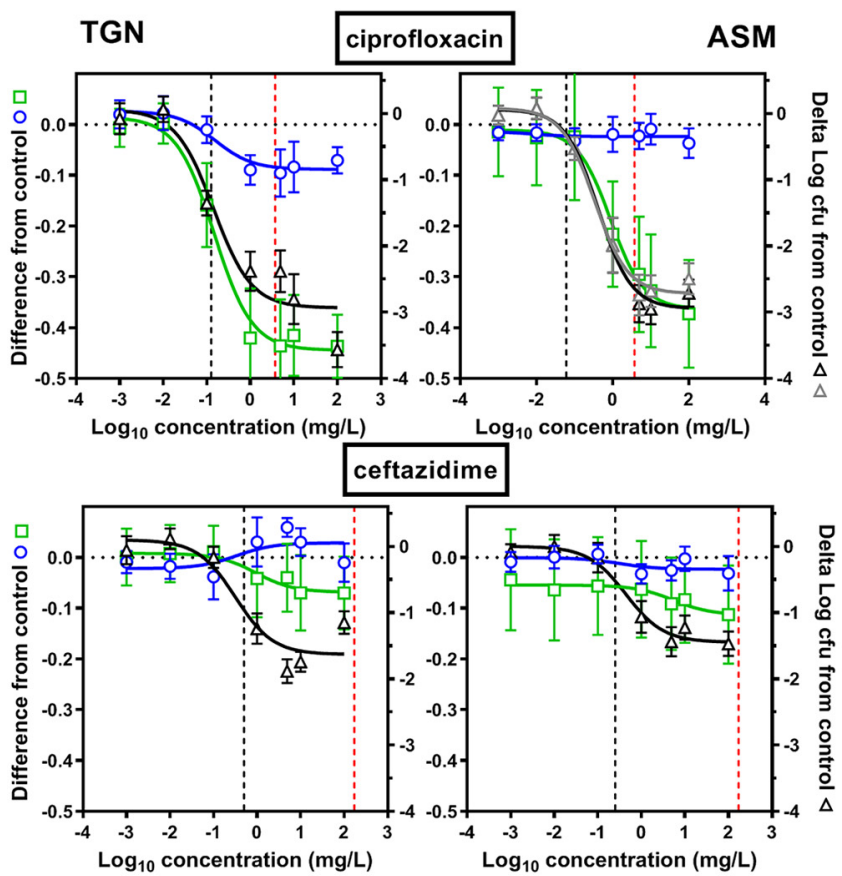

meropenem

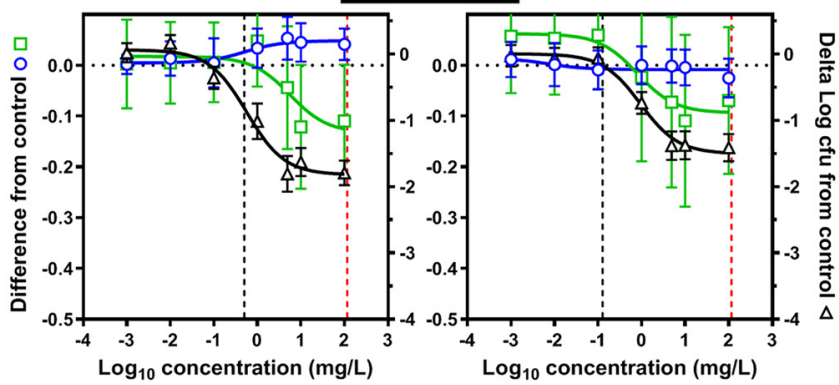

tobramycin

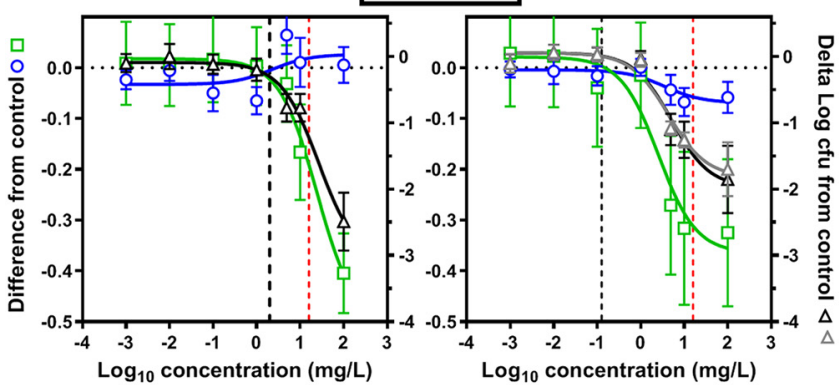

colistin

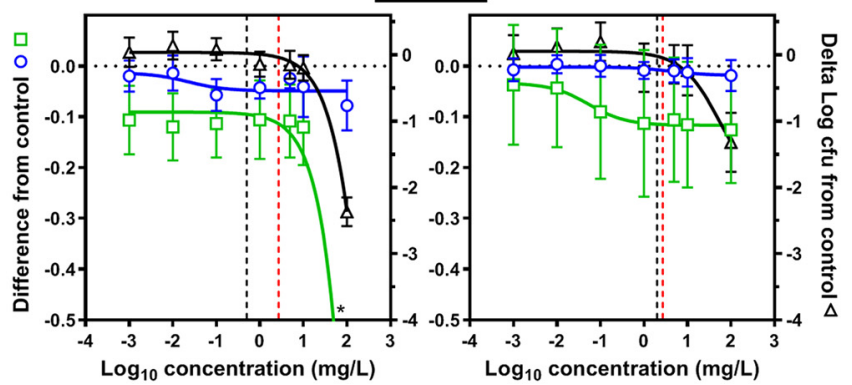

FIG 3 Activities of antibiotics against biofilms in TGN (left) or in ASM(+) (right). Concentration-responses curves of antibiotics against 24-h biofilms of PAO1. Biofilms were incubated with increasing concentra-

(Continued on next page) 

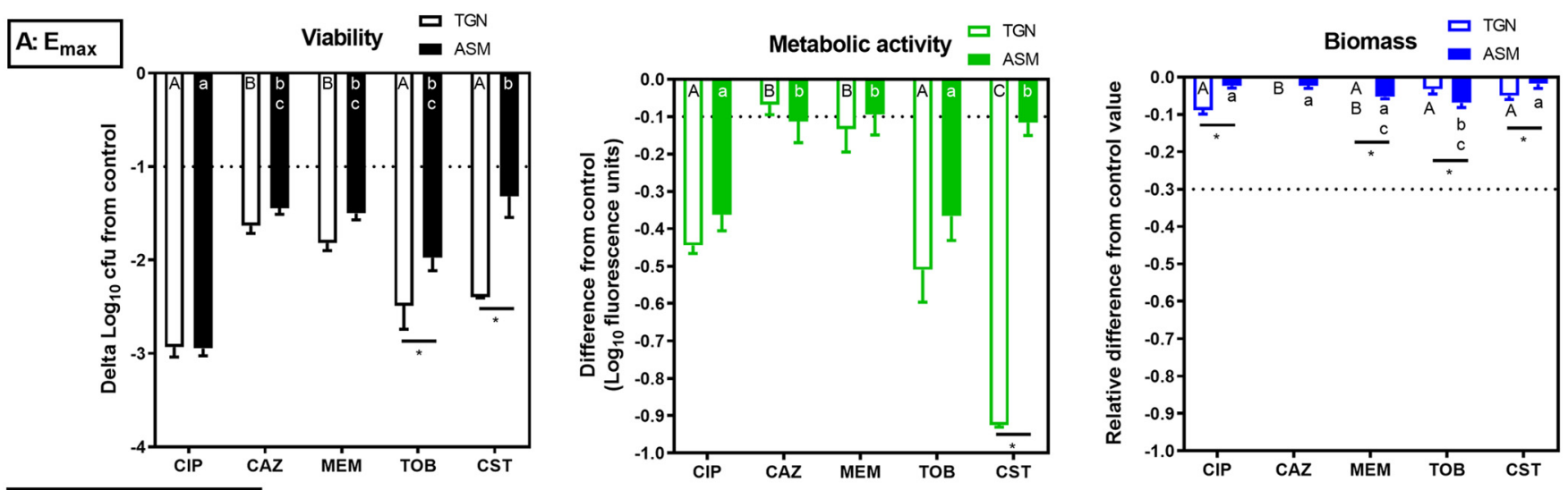

B: relative potencies
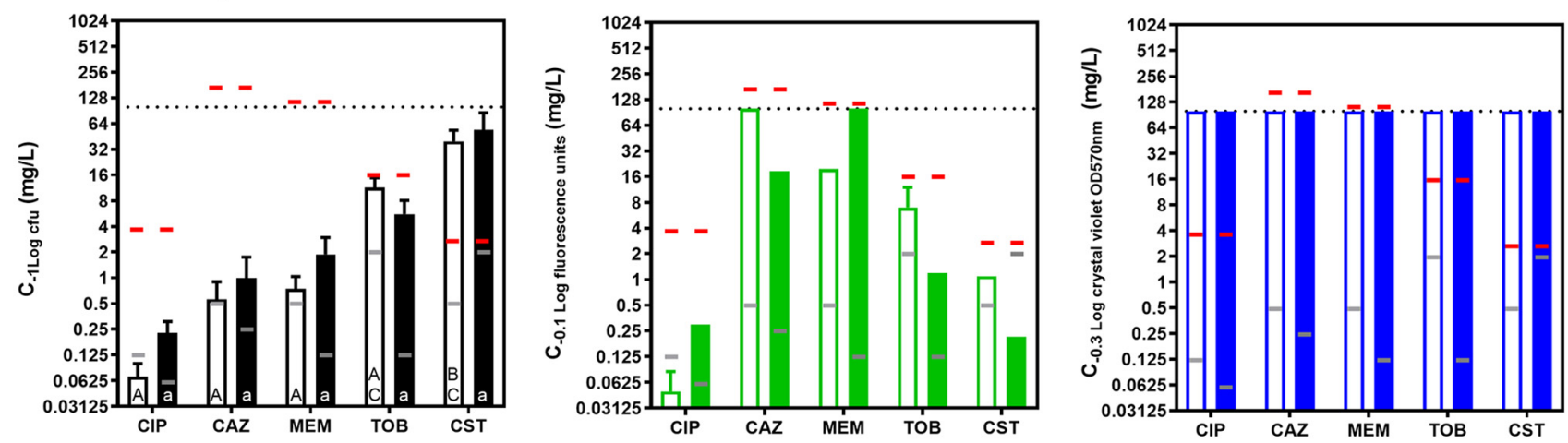

FIG 4 Antibiotic maximal efficacies $\left(E_{\max }\right)(A)$ and antibiotic relative potencies $\left(C_{-1} \log ^{\prime} C_{-0.1}\right.$, or $\left.C_{-0.3}\right)(B)$ compared to the values obtained with no treatment for PAO1 in TGN (open bars) and in ASM(+) (closed bars). (A) The graphs show the maximal reduction in the numbers of CFU (left; viability), in log fluorescein fluorescence (middle; metabolic activity), or in log crystal violet $\mathrm{OD}_{570}$ (right; biofilm mass) compared to the values for the untreated controls and as extrapolated from the sigmoid regression of the concentration-response curve for an infinitely large concentration (Fig. 3). For tobramycin and colistin in TGN and for colistin in $\operatorname{ASM}(+), E_{\max }$ could not be calculated based on the equation of the sigmoidal regression (the plateau was not reached); the graph therefore shows the effect observed at the highest concentration tested. In the other cases, the $E_{\max }$ value is very similar to the effect observed at a concentration of $100 \mathrm{mg} / \mathrm{liter}$, as the plateau was already reached at this concentration. (B) The graphs show the antibiotic concentration (in milligrams per liter) causing a reduction in the number of CFU of $1 \log _{10}$ (left; viability) $\left(C_{-1} \mathrm{log}\right)$, which corresponds to the concentration needed for a 0.1 -log ${ }_{10}$ reduction in fluorescein fluorescence (middle; metabolic activity) $\left(C_{-0.1}\right)$ or a $0.3-\log _{10}$ decrease in the crystal violet $\mathrm{OD}_{570}$ (right; biofilm mass) ( $\left.C_{-0.3}\right)$, compared to the values for the untreated controls and as intrapolated from the sigmoid regression of the concentration-response curve (Fig. 3). These effects are highlighted by the horizontal dotted line in the top row. Red horizontal lines correspond to the human $C_{\max }$ after intravenous administration of a high dose; gray horizontal lines correspond to the MICs in the corresponding medium (Table 1). CIP, ciprofloxacin; CAZ, ceftazidime; MEM, meropenem; TOB, tobramycin; CST, colistin. Values are means \pm SEM from 3 or 4 independent experiments performed in triplicate. Statistical analyses were performed by two-way analysis of variance with Tukey's posttest for multiple comparisons; values with different letters are significantly different from each other $(P<0.05)$. Capital letters compare the results for the antibiotics in TGN; lowercase letters compare the results for the antibiotics in $\mathrm{ASM}(+)$ (statistics could not be performed on $C_{-0.1}$ values [metabolic activity] because the confidence interval on the values was large due to the shape of the curves around this value). ${ }^{*}$, significant difference between media for a given antibiotic.

numbers of CFU in biofilms and planktonic cultures, respectively]; see Fig. S1 in the supplemental material), but the range of concentrations over which a reduction in the fluorescence signal was observed matched that over which the antibiotics reduced the CFU counts.

\section{FIG 3 Legend (Continued)}

tion of antibiotics for $24 \mathrm{~h}$ (ciprofloxacin, ceftazidime, meropenem, tobramycin, and colistin). The left ordinate shows the decrease in metabolic activity (fluorescein assay, green open squares) or biofilm mass (crystal violet assay, blue open circles), expressed as the decrease from the control value (no antibiotic present; $\Delta$ log fluorescence units for the metabolic assay; $\Delta \log \mathrm{OD}_{570}$ for crystal violet staining). The right ordinate shows the change in viability (CFU counts; colonies were allowed to grow for $24 \mathrm{~h}$ [black open triangles] or $48 \mathrm{~h}$ [gray open triangles] on PIA plates), expressed as the reduction (on a log scale) from the control value (the value obtained with no antibiotic present). The vertical black dashed line is the MIC of the antibiotic in the corresponding medium, and the red dashed line is the human $C_{\text {max }}$ after the intravenous administration of a high dose (Table 1). All values are means \pm SEM from 3 or 4 independent experiments performed in triplicate (when not visible, the error bars are smaller than the size of symbols). Note that the last data point is at a value lower than -0.5 for metabolic activity with colistin in TGN $(-0.9$, highlighted by an asterisk), but the scale was maintained to allow comparison with all the other graphs. 

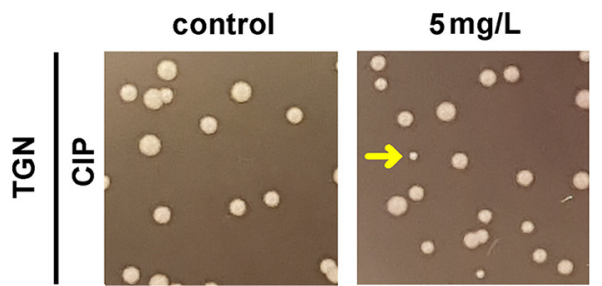

$10 \mathrm{mg} / \mathrm{L}$
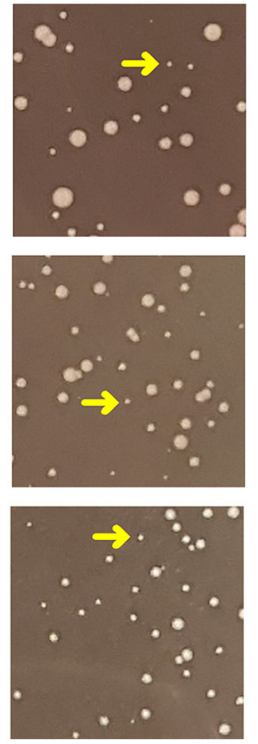

$100 \mathrm{mg} / \mathrm{L}$
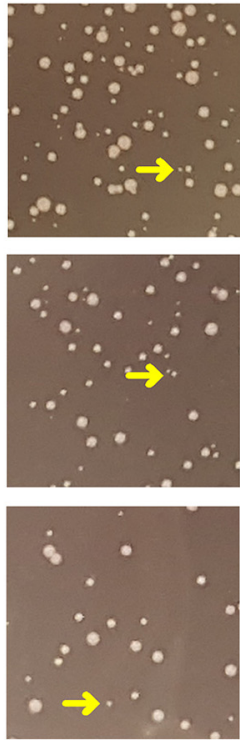

FIG 5 Morphology of colonies of bacteria recovered from biofilms of PAO1 cultivated in TGN medium or $\mathrm{ASM}(+)$ and exposed for $24 \mathrm{~h}$ to ciprofloxacin (CIP) or tobramycin (TOB) at 5, 10, or $100 \mathrm{mg} /$ liter or under control conditions and plated on PIA. The arrows point to typical colonies of small-colony variants.

Regarding biomass, all antibiotics failed to cause a significant decrease in crystal violet absorbance in both media.

Adaptability of the model to CF clinical isolates. Clinical isolates collected from patients with CF markedly differ from PAO1 and are usually considered higher biofilm producers, partially due to their greater twitching motility (18). We therefore examined whether the model that we set up with PAO1 could also be used for CF clinical isolates. To this effect, we selected 4 clinical isolates fully susceptible to the antibiotics that we were testing here (Table 2) and compared them to PAO1 for their twitching motility, their capacity to form biofilms, and their susceptibility to antibiotics in planktonic cultures and biofilms grown in ASM(+). As illustrated in Fig. 6, these isolates showed higher twitching motility than PAO1 (except for isolate 193-2) and formed a well-visible biofilm below the surface of the culture medium. We then quantified the CFU and biomass for biofilms grown in $\operatorname{ASM}(+)$. We did not study the metabolization of fluorescein diacetate for these isolates, because we showed that it was poorly sensitive to the detection of changes in viability after antibiotic exposure for PAO1. Figure 7 shows that, while the CFU counts were similar in clinical isolates and PAO1, biofilm biomass was significantly higher for these isolates than for the reference strain.

In a next step, we examined the activity of the five antibiotics tested previously against these 4 isolates. The MICs in $\mathrm{ASM}(+)$ were similar (in most of the cases, \pm 1 or

TABLE 2 Antibiotic susceptibility of clinical isolates in MHB-ca and ASM(+)

\begin{tabular}{|c|c|c|c|c|c|c|c|c|}
\hline \multirow[b]{3}{*}{ Antibiotic } & \multicolumn{8}{|c|}{ MIC (mg/liter) } \\
\hline & \multicolumn{2}{|c|}{$616($ PA921) } & \multicolumn{2}{|c|}{3807 (PA970) $^{a}$} & \multicolumn{2}{|c|}{ 154-1 (PA1024) ${ }^{b}$} & \multicolumn{2}{|c|}{$193-2(\mathrm{PA} 1060)^{b}$} \\
\hline & MHB-ca & $\operatorname{ASM}(+)^{c}$ & MHB-ca & $\operatorname{ASM}(+)$ & MHB-ca & $\operatorname{ASM}(+)$ & MHB-ca & $\operatorname{ASM}(+)$ \\
\hline Ciprofloxacin & 0.5 & 0.5 & 0.032 & 0.064 & 0.5 & 0.5 & 2 & 2 \\
\hline Ceftazidime & 2 & 0.5 & 1 & 1 & 2 & 0.5 & 2 & 1 \\
\hline Meropenem & 0.125 & 0.064 & 0.125 & 0.064 & 0.5 & 1 & 2 & 4 \\
\hline Tobramycin & 4 & 4 & 2 & 1 & 4 & 8 & 1 & 2 \\
\hline Colistin & 2 & 2 & 2 & 4 & 2 & 4 & 1 & 8 \\
\hline
\end{tabular}

aLaboratoire de Bactériologie, Hôpital Jean Minjoz, Besançon, France.

bUniversity Hospital Münster, Münster, Germany.

cMICs were determined by counting of the number of CFU after plating due to the turbidity of the medium. 


\section{twitching motility}

PAO1
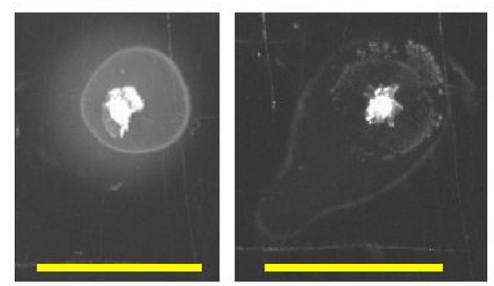

TGN
3807

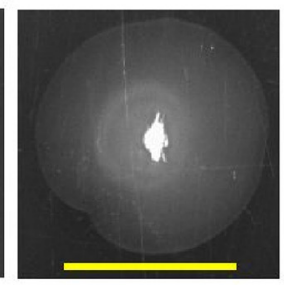

$154-1$

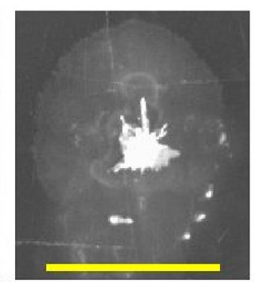

ASM
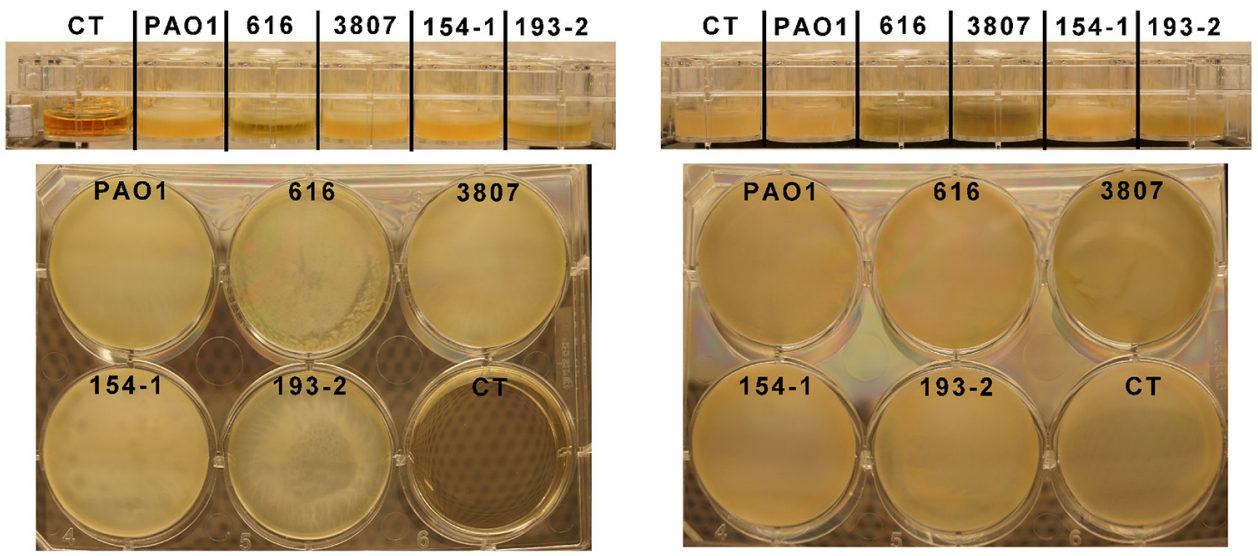

FIG 6 (Top) Twitching motility of PAO1 and of the 4 CF isolates under study. Bars, $1 \mathrm{~cm}$. (Middle and bottom) Lateral view (middle) and bottom view (obtained using a mirror; bottom) of biofilms made by PAO1 and the 4 CF isolates under study in TGN or ASM(+). The biofilms were cultured in 24-well (lateral view) or 6-well (bottom view) plates for $24 \mathrm{~h}$ (TGN) or $24 \mathrm{~h}$ in ASM(-) followed by $24 \mathrm{~h}$ in $\mathrm{ASM}(+)$ (ASM), and pictures were taken under control conditions. The control (CT) is a well containing the culture medium only.

2 doubling dilutions, as for PAO1) to those in MHB-ca (Table 2). We then studied the antibiotic activity against biofilms grown in $\mathrm{ASM}(+)$, focusing on concentrations corresponding to their respective human $C_{\max }$ and on the maximal concentration tested against PAO1 (100 mg/liter). Considering the effects of the antibiotics on viability within the biofilms, ciprofloxacin was the most active and colistin was the least active against all strains, as already reported for PAO1 (Fig. 8, left). Some variations in the reduction in the number of CFU were noticed among isolates for each individual antibiotic. They

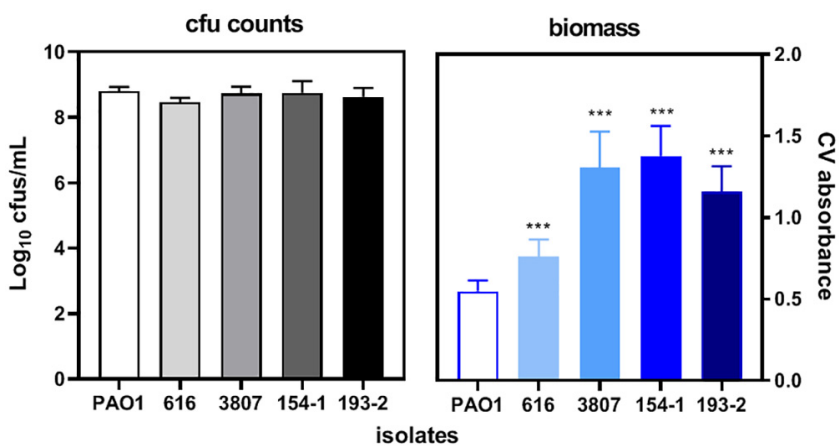

FIG 7 Quantification of CFU (left) and biomass (right; evaluated by crystal violet absorbance) in biofilms made by $4 \mathrm{CF}$ clinical isolates in comparison with those made by PAO1 after $24 \mathrm{~h}$ of growth in $\mathrm{ASM}(-)$ followed by $24 \mathrm{~h}$ of incubation in ASM(+). Data are means \pm SEM from 2 independent experiments in 3 replicates for CFU and from 2 experiments in 2 replicates for biomass. Statistical analyses were performed by 1-way analysis of variance with Dunnett's post hoc test comparing the results for each isolate with those for PAO1. ${ }^{* * *}, P<0.001$. 

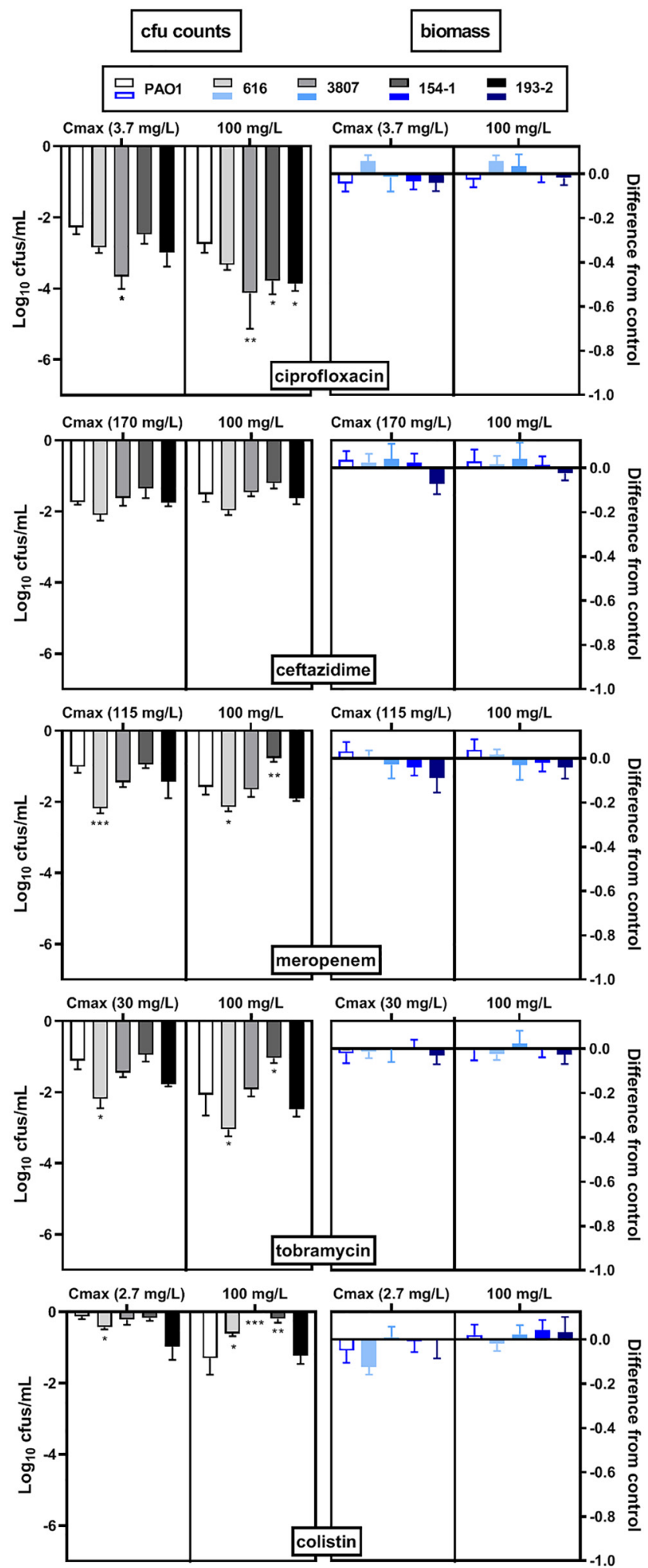

FIG 8 Activity of antibiotics against biofilms grown in $\mathrm{ASM}(+)$ for 4 CF clinical isolates in comparison with that for PAO1 and thereafter incubated with antibiotics (ciprofloxacin, ceftazidime, meropenem, tobramycin, and colistin) at their human $C_{\max }$ (Table 1) or at $100 \mathrm{mg} /$ liter (the maximal concentration tested in the concentration-response curves for PAO1 in Fig. 3) for $24 \mathrm{~h}$. The graphs show the reduction in the $\log _{10}$ number of CFU (left) or in the $\log _{10} O_{570}$ of crystal violet compared to the values for the untreated control. Data are means \pm SEM from 2 independent experiments in 3 replicates for CFU and from 2 experiments in 2 replicates for biomass. Statistical analyses were performed by 1-way analysis of variance with Dunnett's post hoc test comparing each isolate with PAO1. ${ }^{* *}, P<0.001 ;{ }^{* *}, P<0.01 ;{ }^{*}, P<0.05$. 
were the most important for ciprofloxacin at $100 \mathrm{mg} /$ liter, which was significantly more active against 2 out of the 4 isolates than against PAO1 (see Fig. S2 for a picture of the corresponding biofilms). As observed for PAO1, drug effects on biomass were minimal (Fig. 8, right). SCVs were observed under the same conditions observed for PAO1 (not shown).

\section{DISCUSSION}

This study is, to the best of our knowledge, the first one to present a comparative view of the pharmacodynamics of antibiotics against $P$. aeruginosa biofilms in the context of cystic fibrosis. Of interest also, we used here in vitro models comparable to those previously published for Staphylococcus aureus (14), allowing for drawing a point-by-point parallel between these two pathogens that play an important role in lung infections in patients with cystic fibrosis.

A first point of interest is thus to compare the kinetics of biofilm development for both bacterial species. While a plateau value (corresponding to a stable biofilm) was obtained after $24 \mathrm{~h}$ of incubation for $S$. aureus or for $P$. aeruginosa in TGN, we noticed a delay in CFU growth as well as in increase in metabolic activity for $P$. aeruginosa biofilms cultivated in $\operatorname{ASM}(+)$, since the plateau was reached after $48 \mathrm{~h}$ only. We attribute this delay to the need for a 24-h adhesion phase in medium without agar to ensure further $P$. aeruginosa growth. In addition, although the number of CFU was similar for $S$. aureus and $P$. aeruginosa in both media for mature biofilms, the biomass, evaluated by crystal violet staining, was lower for $P$. aeruginosa than for $S$. aureus, as well as in TGN versus $\operatorname{ASM}(+)$. As previously suggested (19), this could possibly be explained by an inadequate fixation of crystal violet to the biofilms, especially in TGN, because of the larger amounts of water present in the rather slimy matrix built up by P. aeruginosa.

Another salient observation concerns the major difference in the rate of metabolization of FDA by $P$. aeruginosa in biofilms versus planktonic cultures. Like Peeters et al. (19), we found a linear relationship between the fluorescence signal and the CFU counts over a range of approximately $10^{5}$ to $10^{8} \mathrm{CFU}$ for both planktonic and biofilmembedded bacteria, but with much higher signals being seen in biofilms at low bacterial counts, suggesting higher metabolic activity under these conditions than in planktonic cultures. Although this observation is counterintuitive if taking into account the sessile character of bacteria in biofilms, it has already been reported and interpreted as denoting higher levels of hydrolytic enzymes in biofilms (20). Corroborating this hypothesis, secreted esterases are known to be overexpressed during biofilm formation by $P$. aeruginosa (21), yet this high rate of conversion of FDA in biofilms considerably reduces the sensitivity and suitability of the method to evaluate antibiotic activity in biofilms, pleading for the systematic use of CFU counting.

Considering these antibiotic effects on CFU counts, we observed maximal efficacies ranging from a 1.5 - to a $3-\log _{10}$ decrease in the numbers of CFU, which is similar to what we previously described for S. aureus in TGN (14). Very few in vitro studies have looked into the details of the pharmacodynamics of antibiotic activity against $P$. aeruginosa in the context of cystic fibrosis, generally concentrating on the determination of minimal biofilm eradication concentration (MBEC) values against clinical isolates. These MBEC have been determined in TSB supplemented with $1 \%$ glucose and found to be 300 - to 600 -fold higher than the MICs for ciprofloxacin, tobramycin, and colistin and more than 2,500-fold higher than the MICs for $\beta$-lactams (22), which is coherent with our finding that the maximal efficacy of $\beta$-lactams is lower than that of the other drugs.

In contrast to our previous observations with S. aureus (14), the only drugs for which we observed a reduction in maximal efficacy in $\mathrm{ASM}(+)$ versus TGN were tobramycin and colistin, i.e., the two drugs which are hydrophilic polycations. This result is coherent with the results of previous studies. First, tobramycin and, to a lesser extent, colistin were found to be more active against biofilms grown in Luria-Bertani broth than against biofilms grown in $\operatorname{ASM}(-)$, but at concentrations higher than those tested here 
(23). These authors attributed this difference to an enrichment of the biofilm matrix in polysaccharides in $\operatorname{ASM}(-)$. This explanation may, however, not apply to our study because TGN is supplemented by glucose, which upregulates the expression of the extracellular polysaccharide-related gene $p s / A$, required for biofilm formation (24). Second, the addition of frozen mucus from patients with CF to preformed biofilms was shown to decrease the activity of tobramycin, which was related to a reduced transport of this polycationic drug in the biofilm (25) due to its strong ionic interactions with components of the biofilm matrix and/or mucus (26). Likewise, colistin strongly binds to mucin in vitro, which considerably decreases its activity (27).

In our previous work with S. aureus, we evidenced SCVs specifically in biofilms cultivated in $\mathrm{ASM}(+)$ and exposed to tobramycin (14). The same observation was made here, but it was extended to ciprofloxacin in both culture media. While the selection of SCVs by aminoglycosides was described a long time ago in different species, including S. aureus (28), P. aeruginosa (29), or Enterobacteriaceae (30), less is known about those selected by fluoroquinolones. They were described in Salmonella enterica as a transient response to stress (31), but also in $P$. aeruginosa biofilms grown in TSB (32) or in ASM(-) (33). No specific auxotrophism could be determined for these SCVs (33). P. aeruginosa SCVs are generally considered high biofilm producers (34), less motile (33) and hyperpiliated (35), to be adapted to oxidative stress (35), and producers of smaller amounts of siderophores (36). They are frequently isolated from the sputum of patients with CF but revert quickly to a normal growing phenotype $(9,37)$. Similar to the SCVs selected in vitro, those isolated from patients do not show any auxotrophism (37). Thus, these SCVs are considered as participating to the physiopathology of biofilm-related infections in the lungs of patients with CF (38).

In contrast to many studies which use extremely high antibiotic concentrations in order to measure MBEC, our concentration-response curves cover the range of concentrations that can be achieved in vivo after intravenous administration. This allows us to conclude that, in both media, a $1-\log _{10}$ decrease in CFU counts could be achieved at concentrations below the human $C_{\max }$ for all drugs except colistin. For the $\beta$-lactams and ciprofloxacin, decreases of approximately $1.5 \log _{10}$ and $3 \log _{10}$, respectively, were obtained at this $C_{\text {max }}$, which would argue in favor of the use of these drugs. Yet tobramycin and colistin are also administered by inhalation, generating higher local concentrations, but it should be mentioned that SCVs were more frequently recovered in the sputum from patients receiving aerosolized antibiotics than in patients receiving intravenous antibiotics (37).

Importantly, the biofilm model that we set up here is applicable to isolates from patients suffering from CF. Of interest, these biofilms all form below the surface of the medium, as previously described, and show the presence of bacterial aggregates similar to those found in the sputum of patients with CF $(13,39)$. We confirm that these isolates globally show a higher twitching motility, recognized as being necessary for exploration and attachment to surfaces and well as for the formation and development of the biofilm architecture (18). They also produce more biofilm biomass than the reference strain. In spite of this larger matrix amount, however, they do not show a lower susceptibility to antibiotics than PAO1, and they even sometimes show a higher susceptibility. Further studies are thus needed to establish the respective role of $\mathrm{ASM}(+)$ components and matrix constituents in limiting antibiotic access to bacteria embedded in biofilms.

Our study suffers from some limitations. We included only a few clinical isolates in our work, which may not represent the diversity encountered in clinical isolates, especially because we selected on purpose only fully susceptible ones. We did not modulate the $\mathrm{pH}$ or the oxygenation of the medium, which may differ from the physiological conditions in the CF lungs, possibly affecting bacterial growth and antibiotic activity $(40,41)$. We exposed our biofilms to fixed antibiotic concentrations and for a specific incubation time, which do not mimic the pharmacokinetic variations observed in patients but keep the advantage of facilitating a direct comparison of pharmacodynamic profiles among drugs. 
Taking these drawbacks into account, our data nevertheless contribute to rationalize therapeutic failures in the treatment of pulmonary infections by $P$. aeruginosa in cystic fibrosis, while our model paves the way to further studies aiming at examining in more detail clinical isolates, including those harboring multiresistance; other drugs or drug combinations; as well as innovative therapeutic options.

\section{MATERIALS AND METHODS}

Bacterial strain and antibiotics. P. aeruginosa PAO1 was used as a reference strain. Four isolates from patients with cystic fibrosis previously characterized to be fully susceptible to the antibiotics used in this study were included in specific experiments (42). These strains were routinely grown on Trypticase soy agar (TSA; VWR, Radnor, PA) for overnight culture and on Pseudomonas isolation agar (PIA; Sigma-Aldrich, St. Louis, MO) for determination of the numbers of CFU (colonies were easier to count [round shape] and contaminations could be excluded on this medium).

Antibiotics were obtained as microbiological standards as follows: ciprofloxacin $\mathrm{HCl}$ (potency, 93.9\%) was from Bayer (Leverkusen, Germany), tobramycin (potency, 100\%) was from Galephar (Marche-enFamenne, Belgium), ceftazidime pentahydrated (potency, 72.5\%) was from Panpharma (LuitréDompierre, France), and colistin sulfate salt (potency, 79.6\%) was from Sigma-Aldrich. Meropenem (potency, 74\%) was procured as a generic drug branded product distributed for human parenteral use in Belgium by Sandoz (Holzkirchen, Germany).

Culture media for biofilm cultures. Three media were used in parallel, namely, Trypticase soy broth (VWR) supplemented with $1 \%$ glucose and $2 \% \mathrm{NaCl}(\mathrm{TGN})(17,43)$ and two types of artificial sputum media (adapted from references 11,44, and 45), differing by the fact one is supplemented in agar $[\operatorname{ASM}(+)]$ and the other one is not $[\mathrm{ASM}(-)]$. ASM(-) contains, per liter, $10 \mathrm{~g}$ mucin (Sigma-Aldrich), 4 g DNA (Sigma-Aldrich), $5.9 \mathrm{mg}$ diethylenetriaminepentaacetic acid (DTPA; Sigma-Aldrich), $5 \mathrm{~g} \mathrm{NaCl}$ (VWR), $2.2 \mathrm{~g} \mathrm{KCl}$ (Sigma-Aldrich), $5 \mathrm{~g}$ amino acids (Becton, Dickinson, Franklin Lakes, NJ), $1.81 \mathrm{~g}$ Tris (Calbiochem, San Diego, CA), and $5 \mathrm{ml}$ egg yolk emulsion (Sigma-Aldrich). ASM(+) contains, in addition, $3 \mathrm{~g} /$ liter agar (Becton, Dickinson). All constituents except egg yolk emulsion were autoclaved (egg yolk emulsion was added aseptically to the autoclaved medium), after which the $\mathrm{pH}$ was adjusted to 7 with $\mathrm{NaOH}$. We previously showed that $\mathrm{ASM}(+)$ better mimics the composition and viscoelastic properties of the mucus found in the respiratory tract of patients with cystic fibrosis (14).

Macroscopic twitching assay. Twitching motility was determined as previously described (46). Briefly, bacteria were plated onto $1.5 \%$ lysogeny broth (LB) agar plates (prepared using lysogeny broth [Alfa Aesar, Ward Hill, MA] and $1.5 \%$ agar) and incubated overnight at $37^{\circ} \mathrm{C}$, after which a small portion of the bacterial streak was collected with a toothpick and stabbed in the center of a $1 \%$ LB agar plate, which was then incubated at $37^{\circ} \mathrm{C}$ for $24 \mathrm{~h}$. To better visualize the twitching motility, the plates were flooded with a small volume of cold twitching motility (TM) developer solution ( $400 \mathrm{ml}$ deionized water, $100 \mathrm{ml}$ glacial acetic acid [Merck-Millipore, Burlington, MA], $500 \mathrm{ml}$ methanol [Merck-Millipore], stored at $4^{\circ} \mathrm{C}$ ) for $30 \mathrm{~min}$, after which the top colonies were gently scraped with a plastic disposable loop. The TM developer solution and scraped colonies were carefully decanted into a beaker, and images of the plates were taken using a Bio-Rad Molecular imager (Gel Doc XR+ system; Bio-Rad, Hercules, CA).

Development of the biofilm model. Except when stated otherwise, biofilms were grown in 96-well plates (European catalog number 655 180; VWR) as previously described (14) when using TGN but with some modifications in ASM(+). In brief, a bacterial suspension was prepared in cation-adjusted MuellerHinton broth (MHB-ca; Sigma-Aldrich) starting from overnight cultures on TSA. When using TGN for biofilm growth, the 96 -well plates were inoculated (200 $\mu \mathrm{l} /$ well) at approximately $3 \times 10^{7} \mathrm{CFU} / \mathrm{ml}$ (the $\mathrm{OD}_{620}$ was adjusted to 0.05 ) and then incubated at $37^{\circ} \mathrm{C}$ for $24 \mathrm{~h}$ (unless stated otherwise) to obtain a mature biofilm. When ASM(+) was used, $200 \mu \mathrm{l}$ of a suspension at $5.5 \times 10^{7} \mathrm{CFU} / \mathrm{ml}$ in ASM(-) was inoculated in the 96-well plates and the plates were incubated for $24 \mathrm{~h}$ at $37^{\circ} \mathrm{C}$ to allow for bacterial attachment, after which the medium was removed and replaced by $200 \mu \mathrm{l}$ of $\mathrm{ASM}(+)$ and the plates were reincubated for $24 \mathrm{~h}$ at $37^{\circ} \mathrm{C}$ (unless stated otherwise). In all cases, the plates were covered by Breathe-Easy sealing membranes, which are permeable to gas but which limit evaporation (SigmaAldrich). All changes of media or washing steps were carefully performed by slow pipetting from the bottom and close to the edge of the well to avoid removing biofilms growing below the surface of the medium.

Susceptibility testing. MICs were determined by microdilution following the guidelines of the Clinical and Laboratory Standards Institute using cation-adjusted Mueller-Hinton broth (MHB-ca) (47) and compared to those measured in TGN and $\mathrm{ASM}(+)$ following the same protocol. As previously described (14), direct visual reading of the MICs was not possible in $A S M(+)$ due to the intrinsic turbidity of this medium. Aliquots were therefore spread on PIA and incubated overnight, with MICs being defined as the lowest concentration for which there was no change in the number of CFU compared to the initial values. As bacteria tended to form aggregates in $\mathrm{ASM}(+)$, the content of each well was carefully homogenized by back-and-forth pipetting before taking aliquots, diluting them, and spreading them on agar plates.

Activity of antibiotics against biofilms. After reaching maturity $\{24 \mathrm{~h}$ for TGN, $48 \mathrm{~h}$ for ASM $[24 \mathrm{~h}$ in $\operatorname{ASM}(-)$, followed by $24 \mathrm{~h}$ in $\mathrm{ASM}(+)]\}$, the culture medium of the biofilms was removed and replaced with fresh medium (control) or medium supplemented with antibiotics at concentrations ranging from $10^{-3}$ to $10^{2} \mathrm{mg} /$ liter in order to obtain full concentration-response curves. The biofilms were reincubated for $24 \mathrm{~h}$ at $37^{\circ} \mathrm{C}$. At the end of the incubation period, the medium was removed and the biofilm was washed once with $200 \mu \mathrm{l}$ of 3-morpholinopropane-1-sulfonic acid (MOPS) buffer (20.9 g/liter of MOPS [Sigma-Aldrich], $5.6 \mathrm{~g} /$ liter $\mathrm{NaCl}$; the $\mathrm{pH}$ was adjusted to 7 with $\mathrm{NaOH}$ ) (19). Biofilm biomass was 
quantified using crystal violet, a cationic dye that nonspecifically stains negatively charged constituents in biofilms. The washed biofilms were fixed by heat at $60^{\circ} \mathrm{C}$ for about $24 \mathrm{~h}$ and incubated for $10 \mathrm{~min}$ at room temperature with $200 \mu \mathrm{l}$ of crystal violet (final concentration, $0.2 \mathrm{~g} / \mathrm{liter}$; VWR). After removing the excess crystal violet, the plates were washed under running water and dried. The dye fixed to the biofilm was resolubilized in $200 \mu \mathrm{l}$ of $66 \%$ acetic acid and incubated for $1 \mathrm{~h}$ at room temperature. The absorbance at $570 \mathrm{~nm}$ was measured using a SPECTRAmax Gemini XS microplate spectrophotometer (Molecular Devices LLC, Sunnyvale, CA) (14). The metabolic activity (vitality) in the biofilms was quantified using the fluorescein diacetate (FDA) assay. It is based on the hydrolysis by living bacteria of the nonfluorescent white dye fluorescein diacetate in the yellow highly fluorescent fluorescein (19). This dye is preferred to resazurin (which we used in $S$. aureus biofilms) to evaluate the metabolic activity of $P$. aeruginosa because it generates much higher fluorescence signals (19). The washed biofilms were incubated with $100 \mathrm{mg} /$ /iter fluorescein diacetate (Sigma-Aldrich) for $15 \mathrm{~min}$ (unless stated otherwise) at $37^{\circ} \mathrm{C}$ in the dark. Fluorescein fluorescence was measured at a wavelength of $518 \mathrm{~nm}$ with an excitation wavelength of $494 \mathrm{~nm}$ using a SPECTRAmax Gemini XS microplate spectrofluorometer. Bacterial counts were determined in washed biofilms resuspended in $1 \mathrm{ml}$ of sterile phosphate-buffered saline in microcentrifugation tubes. The tubes were vortexed, placed for $5 \mathrm{~min}$ in a sonication bath (Bransonic Ultrasonic cleaner $3510 \mathrm{E}-\mathrm{MT}$; frequency, $40 \mathrm{kHz}$; Danbury, CT) to disrupt the biofilm, and vortexed again, after which aliquots were taken and diluted before spreading on PIA plates. Unless stated otherwise, the colonies were counted after $24 \mathrm{~h}$ of incubation at $37^{\circ} \mathrm{C}$.

Curve-fitting and statistical analyses. Curve-fitting analyses were made using GraphPad Prism (version 8.02) software (GraphPad Software, San Diego, CA). Data were used to fit a sigmoid function, which allowed us to calculate the maximal effect $\left(E_{\max } ;\right.$ the maximal reduction in viability, vitality, or biomass for an infinitely large concentration of antibiotic) and relative potencies (the antibiotic concentration needed for a reduction in the number of CFU in the biofilms of $1 \log _{10}\left[C_{-1 / \mathrm{log}}\right]$, which corresponds to the concentration needed for a $0.1-\log _{10}$ reduction in fluorescein fluorescence [vitality] $\left[C_{-0.1}\right]$ or a $0.3-\log _{10}$ decrease in the crystal violet $\mathrm{OD}_{570}$ [biomass] [ $\left.\left.C_{-0.3}\right]\right)$. Statistical analyses were performed with GraphPad Instat (version 3.06) software (GraphPad Software) or GraphPad Prism (version 8.02) software.

\section{SUPPLEMENTAL MATERIAL}

Supplemental material is available online only.

SUPPLEMENTAL FILE 1, PDF file, 0.5 MB.

\section{ACKNOWLEDGMENTS}

Y.D.I. received a Ph.D. fellowship from the Belgian Fonds pour la Recherche dans I'Industrie et l'Agriculture (FRIA), followed by a bourse du patrimoine of the Université catholique de Louvain. This work was supported by the FNRS (grants T.0189.16, J.0018.17, and J.0162.19) and the Fonds Spéciaux de Recherche from the UCLouvain.

F.V.B. is research director from the Fonds National belge de la Recherche scientifique (FRS-FNRS).

We are grateful to A. Mangin, A. Chaniotacos, and V. Yfantis for helpful technical assistance.

\section{REFERENCES}

1. Cystic Fibrosis Foundation. 2018. 2017 patient registry annual data report. https://www.cff.org/Research/Researcher-Resources/Patient -Registry/2017-Patient-Registry-Annual-Data-Report.pdf. Last updated 1 August 2018. Accessed 16 November 2018.

2. Bjarnsholt T, Jensen PO, Fiandaca MJ, Pedersen J, Hansen CR, Andersen CB, Pressler T, Givskov M, Hoiby N. 2009. Pseudomonas aeruginosa biofilms in the respiratory tract of cystic fibrosis patients. Pediatr Pulmonol 44:547-558. https://doi.org/10.1002/ppul.21011.

3. Ciofu O, Tolker-Nielsen T. 2019. Tolerance and resistance of Pseudomonas aeruginosa biofilms to antimicrobial agents - how $P$. aeruginosa can escape antibiotics. Front Microbiol 10:913. https://doi.org/10.3389/fmicb .2019.00913.

4. Donlan RM, Costerton JW. 2002. Biofilms: survival mechanisms of clinically relevant microorganisms. Clin Microbiol Rev 15:167-193. https:// doi.org/10.1128/cmr.15.2.167-193.2002.

5. Mann EE, Wozniak DJ. 2012. Pseudomonas biofilm matrix composition and niche biology. FEMS Microbiol Rev 36:893-916. https://doi.org/10 .1111/j.1574-6976.2011.00322.x.

6. Ciofu O, Rojo-Molinero E, Macià MD, Oliver A. 2017. Antibiotic treatment of biofilm infections. APMIS 125:304-319. https://doi.org/10.1111/apm .12673.

7. Ciofu O, Tolker-Nielsen T, Jensen PØ, Wang H, Høiby N. 2015. Antimicrobial resistance, respiratory tract infections and role of biofilms in lung infections in cystic fibrosis patients. Adv Drug Deliv Rev 85:7-23. https:// doi.org/10.1016/j.addr.2014.11.017.

8. Cohen TS, Prince A. 2012. Cystic fibrosis: a mucosal immunodeficiency syndrome. Nat Med 18:509-519. https://doi.org/10.1038/nm.2715.

9. Evans TJ. 2015. Small colony variants of Pseudomonas aeruginosa in chronic bacterial infection of the lung in cystic fibrosis. Future Microbiol 10:231-239. https://doi.org/10.2217/fmb.14.107.

10. Malone JG. 2015. Role of small colony variants in persistence of Pseudomonas aeruginosa infections in cystic fibrosis lungs. Infect Drug Resist 8:237-247. https://doi.org/10.2147/IDR.S68214.

11. Sriramulu DD, Lunsdorf H, Lam JS, Romling U. 2005. Microcolony formation: a novel biofilm model of Pseudomonas aeruginosa for the cystic fibrosis lung. J Med Microbiol 54:667-676. https://doi.org/10 .1099/jmm.0.45969-0.

12. Hoiby N, Bjarnsholt T, Moser C, Jensen PO, Kolpen M, Qvist T, Aanaes K, Pressler T, Skov M, Ciofu O. 2017. Diagnosis of biofilm infections in cystic fibrosis patients. APMIS 125:339-343. https://doi.org/10.1111/apm.12689.

13. Kirchner S, Fothergill JL, Wright EA, James CE, Mowat E, Winstanley C. 2012. Use of artificial sputum medium to test antibiotic efficacy against Pseudomonas aeruginosa in conditions more relevant to the cystic fibrosis lung. J Vis Exp 2012:e3857. https://doi.org/10.3791/3857.

14. Diaz Iglesias Y, Wilms T, Vanbever R, Van Bambeke F. 2019. Activity of antibiotics against Staphylococcus aureus in an in vitro model of bio- 
films in the context of cystic fibrosis: influence of the culture medium. Antimicrob Agents Chemother 63:e00602-19. https://doi.org/10.1128/ AAC.00602-19.

15. Palmer KL, Aye LM, Whiteley M. 2007. Nutritional cues control Pseudomonas aeruginosa multicellular behavior in cystic fibrosis sputum. J Bacteriol 189: 8079-8087. https://doi.org/10.1128/JB.01138-07.

16. Turner KH, Wessel AK, Palmer GC, Murray JL, Whiteley M. 2015. Essential genome of Pseudomonas aeruginosa in cystic fibrosis sputum. Proc Natl Acad Sci U S A 112:4110-4115. https://doi.org/10 .1073/pnas.1419677112.

17. Bauer J, Siala W, Tulkens PM, Van Bambeke F. 2013. A combined pharmacodynamic quantitative and qualitative model reveals the potent activity of daptomycin and delafloxacin against Staphylococcus aureus biofilms. Antimicrob Agents Chemother 57:2726-2737. https://doi.org/ 10.1128/AAC.00181-13.

18. Burrows LL. 2012. Pseudomonas aeruginosa twitching motility: type IV pili in action. Annu Rev Microbiol 66:493-520. https://doi.org/10.1146/ annurev-micro-092611-150055.

19. Peeters E, Nelis HJ, Coenye T. 2008. Comparison of multiple methods for quantification of microbial biofilms grown in microtiter plates. J Microbiol Methods 72:157-165. https://doi.org/10.1016/j.mimet.2007.11.010.

20. Tribedi P, Gupta AD, Sil AK. 2015. Adaptation of Pseudomonas sp. AKS2 in biofilm on low-density polyethylene surface: an effective strategy for efficient survival and polymer degradation. Bioresour Bioprocess 2:14. https://doi.org/10.1186/s40643-015-0044-x.

21. Tielen $P$, Rosenau $F$, Wilhelm S, Jaeger KE, Flemming HC, Wingender J. 2010. Extracellular enzymes affect biofilm formation of mucoid Pseudomonas aeruginosa. Microbiology 156:2239-2252. https://doi.org/10 .1099/mic.0.037036-0.

22. Dosler S, Karaaslan E. 2014. Inhibition and destruction of Pseudomonas aeruginosa biofilms by antibiotics and antimicrobial peptides. Peptides 62:32-37. https://doi.org/10.1016/j.peptides.2014.09.021.

23. Rozenbaum RT, van der Mei HC, Woudstra W, de Jong ED, Busscher HJ, Sharma PK. 2019. Role of viscoelasticity in bacterial killing by antimicrobials in differently grown Pseudomonas aeruginosa biofilms. Antimicrob Agents Chemother 63:e01972-18. https://doi.org/10.1128/AAC.01972-18.

24. She P, Wang Y, Liu Y, Tan F, Chen L, Luo Z, Wu Y. 2019. Effects of exogenous glucose on Pseudomonas aeruginosa biofilm formation and antibiotic resistance. Microbiologyopen 8:e933. https://doi.org/10.1002/ mbo3.933.

25. Muller L, Murgia X, Siebenburger L, Borger C, Schwarzkopf K, Sewald K, Haussler S, Braun A, Lehr CM, Hittinger M, Wronski S. 2018. Human airway mucus alters susceptibility of Pseudomonas aeruginosa biofilms to tobramycin, but not colistin. J Antimicrob Chemother 73:2762-2769. https://doi.org/10.1093/jac/dky241.

26. Tseng BS, Zhang W, Harrison JJ, Quach TP, Song JL, Penterman J, Singh PK, Chopp DL, Packman Al, Parsek MR. 2013. The extracellular matrix protects Pseudomonas aeruginosa biofilms by limiting the penetration of tobramycin. Environ Microbiol 15:2865-2878. https://doi.org/10.1111/ 1462-2920.12155.

27. Huang JX, Blaskovich MAT, Pelingon R, Ramu S, Kavanagh A, Elliott AG, Butler MS, Montgomery AB, Cooper MA. 2015. Mucin binding reduces colistin antimicrobial activity. Antimicrob Agents Chemother 59: 5925-5931. https://doi.org/10.1128/AAC.00808-15.

28. Schaaff F, Bierbaum G, Baumert N, Bartmann P, Sahl HG. 2003. Mutations are involved in emergence of aminoglycoside-induced small colony variants of Staphylococcus aureus. Int J Med Microbiol 293:427-435. https://doi.org/10.1078/1438-4221-00282.

29. Gerber AU, Craig WA. 1982. Aminoglycoside-selected subpopulations of Pseudomonas aeruginosa: characterization and virulence in normal and leukopenic mice. J Lab Clin Med 100:671-681.

30. Musher DM, Baughn RE, Merrell GL. 1979. Selection of small-colony variants of Enterobacteriaceae by in vitro exposure to aminoglycosides: pathogenicity for experimental animals. J Infect Dis 140:209-214. https://doi.org/10.1093/infdis/140.2.209.

31. Drescher SPM, Gallo SW, Ferreira PMA, Ferreira CAS, de Oliveira SD. 2019. Salmonella enterica persister cells form unstable small colony variants after in vitro exposure to ciprofloxacin. Sci Rep 9:7232. https://doi.org/ 10.1038/s41598-019-43631-7.

32. Belfield K, Bayston R, Hajduk N, Levell G, Birchall JP, Daniel M. 2017.
Evaluation of combinations of putative anti-biofilm agents and antibiotics to eradicate biofilms of Staphylococcus aureus and Pseudomonas aeruginosa. J Antimicrob Chemother 72:2531-2538. https://doi.org/10 $.1093 / j a c / d k x 192$.

33. Sousa AM, Monteiro R, Pereira MO. 2018. Unveiling the early events of Pseudomonas aeruginosa adaptation in cystic fibrosis airway environment using a long-term in vitro maintenance. Int J Med Microbiol 308:1053-1064. https://doi.org/10.1016/j.ijmm.2018.10.003.

34. Kirisits MJ, Prost L, Starkey M, Parsek MR. 2005. Characterization of colony morphology variants isolated from Pseudomonas aeruginosa biofilms. Appl Environ Microbiol 71:4809-4821. https://doi.org/10.1128/ AEM.71.8.4809-4821.2005.

35. von Gotz F, Haussler S, Jordan D, Saravanamuthu SS, Wehmhoner D, Strussmann A, Lauber J, Attree I, Buer J, Tummler B, Steinmetz I. 2004. Expression analysis of a highly adherent and cytotoxic small colony variant of Pseudomonas aeruginosa isolated from a lung of a patient with cystic fibrosis. J Bacteriol 186:3837-3847. https://doi.org/10.1128/ JB.186.12.3837-3847.2004.

36. Sabra W, Haddad AM, Zeng AP. 2014. Comparative physiological study of the wild type and the small colony variant of Pseudomonas aeruginosa 20265 under controlled growth conditions. World J Microbiol Biotechnol 30:1027-1036. https://doi.org/10.1007/s11274-013-1521-z.

37. Haussler S, Tummler B, Weissbrodt H, Rohde M, Steinmetz I. 1999. Small-colony variants of Pseudomonas aeruginosa in cystic fibrosis. Clin Infect Dis 29:621-625. https://doi.org/10.1086/598644.

38. Drenkard E, Ausubel FM. 2002. Pseudomonas biofilm formation and antibiotic resistance are linked to phenotypic variation. Nature 416: 740-743. https://doi.org/10.1038/416740a.

39. Haley CL, Colmer-Hamood JA, Hamood AN. 2012. Characterization of biofilm-like structures formed by Pseudomonas aeruginosa in a synthetic mucus medium. BMC Microbiol 12:181. https://doi.org/10.1186/ 1471-2180-12-181.

40. Pompilio A, Crocetta V, Pomponio S, Fiscarelli E, Bonaventura GD. 2015 In vitro activity of colistin against biofilm by Pseudomonas aeruginosa is significantly improved under "cystic fibrosis-like" physicochemical conditions. Diagn Microbiol Infect Dis 82:318-325. https://doi.org/10.1016/ j.diagmicrobio.2015.01.006.

41. Quinn RA, Comstock W, Zhang T, Morton JT, da Silva R, Tran A, Aksenov A, Nothias LF, Wangpraseurt D, Melnik AV, Ackermann G, Conrad D, Klapper I, Knight R, Dorrestein PC. 2018. Niche partitioning of a pathogenic microbiome driven by chemical gradients. Sci Adv 4:eaau1908. https://doi.org/10.1126/sciadv.aau1908.

42. Mustafa $M H$, Chalhoub $H$, Denis O, Deplano A, Vergison A, RodriguezVillalobos H, Tunney MM, Elborn JS, Kahl BC, Traore H, Vanderbist F, Tulkens PM, Van Bambeke F. 2016. Antimicrobial susceptibility of Pseudomonas aeruginosa isolated from cystic fibrosis patients in northern Europe. Antimicrob Agents Chemother 60:6735-6741. https://doi.org/ 10.1128/AAC.01046-16.

43. Siala W, Mingeot-Leclercq MP, Tulkens PM, Hallin M, Denis O, Van Bambeke F. 2014. Comparison of the antibiotic activities of daptomycin, vancomycin, and the investigational fluoroquinolone delafloxacin against biofilms from Staphylococcus aureus clinical isolates. Antimicrob Agents Chemother 58: 6385-6397. https://doi.org/10.1128/AAC.03482-14.

44. Ghani M, Soothill JS. 1997. Ceftazidime, gentamicin, and rifampicin, in combination, kill biofilms of mucoid Pseudomonas aeruginosa. Can J Microbiol 43:999-1004. https://doi.org/10.1139/m97-144.

45. Tavernier S, Coenye T. 2015. Quantification of Pseudomonas aeruginosa in multispecies biofilms using PMA-qPCR. PeerJ 3:e787. https://doi.org/ 10.7717/peerj.787.

46. Turnbull L, Whitchurch CB. 2014. Motility assay: twitching motility. Methods Mol Biol 1149:73-86. https://doi.org/10.1007/978-1-4939-0473-0_9.

47. Clinical and Laboratory Standards Institute. 2018. Performance standards for antimicrobial susceptibility testing; 28th informational supplement. CLSI document M100-S28. Clinical and Laboratory Standards Institute, Wayne, PA.

48. Karaiskos I, Friberg LE, Pontikis K, loannidis K, Tsagkari V, Galani L, Kostakou E, Baziaka F, Paskalis C, Koutsoukou A, Giamarellou H. 2015. Colistin population pharmacokinetics after application of a loading dose of $9 \mathrm{MU}$ colistin methanesulfonate in critically ill patients. Antimicrob Agents Chemother 59:7240-7248. https://doi.org/10.1128/AAC.00554-15. 
Activity of antibiotics against Pseudomonas aeruginosa in an in vitro model of biofilms in the context of cystic fibrosis : influence of the culture medium.

Yvan Diaz Iglesias, ${ }^{1}$ Françoise Van Bambeke. ${ }^{1{ }^{*}}$

${ }^{1}$ Pharmacologie cellulaire et moléculaire; Louvain Drug Research Institute, Université catholique de Louvain (UCLouvain), Brussels, Belgium

Supplementary material 
Figure S1. Fluorescein fluorescence signal measured for PAO1 at increasing inocula after 15 minutes of incubation with $100 \mathrm{mg} / \mathrm{L}$ fluorescein diacetate (FDA). The graphs compare data from planktonic cultures in MHB-ca (same curve on both graphs; this curve is also shown in Figure 1) and from biofilms grown in TGN (left) or in ASM (right). In biofilms, the bacterial counts were those recovered from biofilms incubated with antibiotics at different concentrations and FDA fluorescence values were those measured in independent wells exposed to the same treatments. The linear regression of the data obtained in biofilms are shown on each graph. The slope is approx. 0.1 in both media, meaning that increasing of $1 \log _{10}$ cfus generates a $0.1 \log _{10}$ increase in fluorescein fluorescence signal.

FDA metabolisation: comparison planktonic cultures vs biofilms inTGN

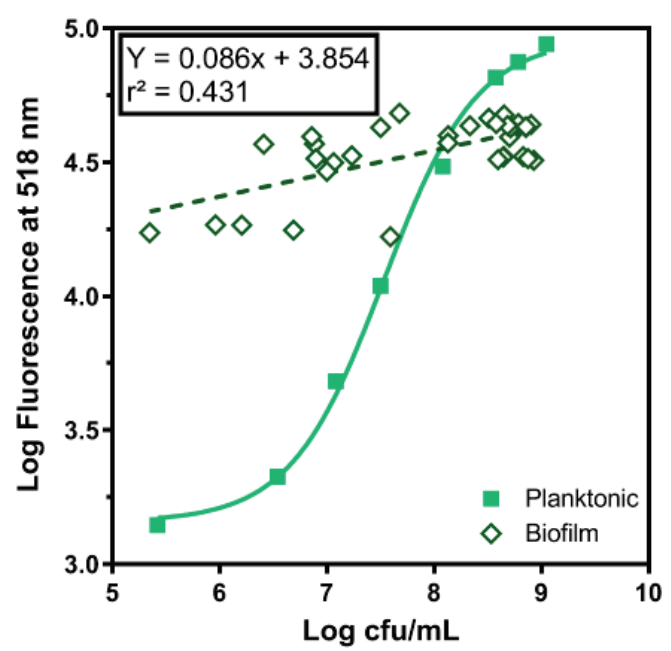

FDA metabolisation:

comparison planktonic cultures vs biofilms in ASM

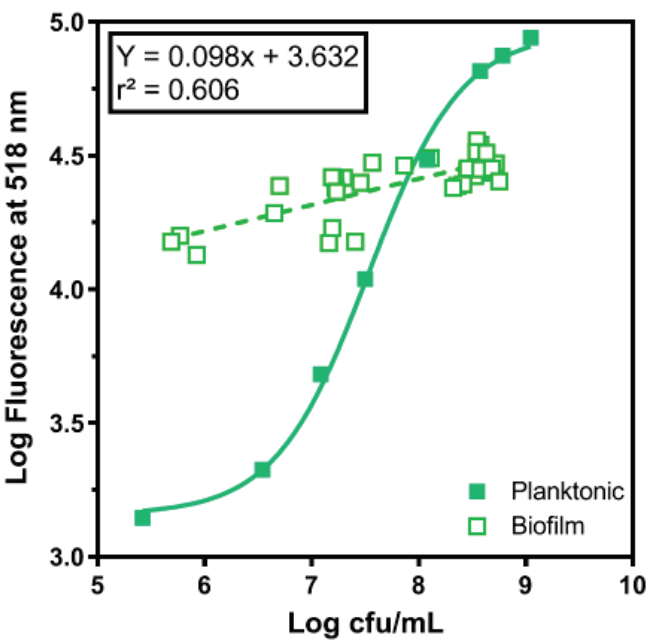


Figure S2 : lateral view of biofilms of PAO1 and $4 \mathrm{CF}$ clinical isolates grown during $24 \mathrm{~h}$ in $\mathrm{ASM}(-)$, then $24 \mathrm{~h}$ in $\mathrm{ASM}(+)$, and incubated during $24 \mathrm{~h}$ additional hours in control conditions (no antibiotic added) or in the presence of ciprofloxacin at $100 \mathrm{mg} / \mathrm{L}$ or at its human $\mathrm{C}_{\max }(3.7$ $\mathrm{mg} / \mathrm{L})$.

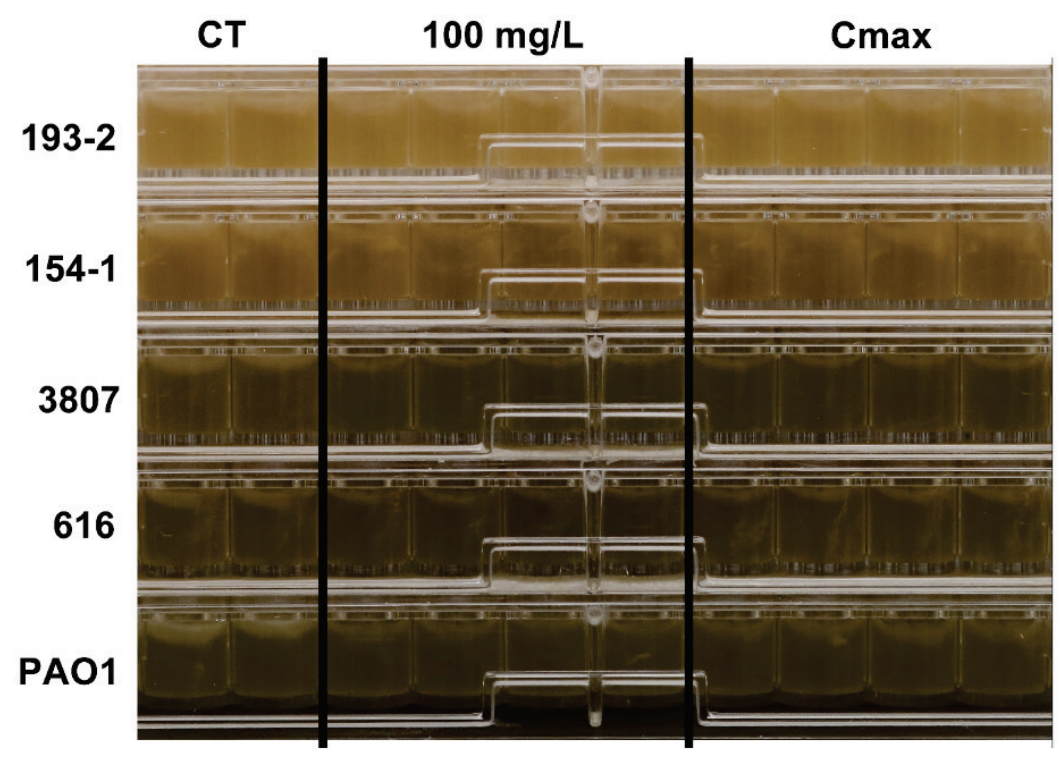

